\title{
Relación entre supervisión parental $y$ conducta antisocial en menores infractores del Estado de Morelos
}

\author{
Lorena Carrillo Amezcua, ${ }^{1}$ Francisco Juárez García, ${ }^{1}$ Catalina González-Forteza, ${ }^{1}$ Nora Angélica Martínez \\ Vélez, ${ }^{1}$ María Elena Medina-Mora Icaza ${ }^{2}$
}

Artículo original

\section{ABSTRACT}

\section{Introduction}

Parental supervision is constituted by a series of correlated parental behaviors involving children care, knowing their where abouts, which activities they are performing, and the situation of their adaptive processes. In order for parental supervision to take place, it is necessary that communication and mutual support exist between mother and father, as well as between parents and children so that the family environment becomes pleasant. Supervision is one of the most studied factors related to anti-social behavior, and it has been observed that its absence or inconsistency allows for the occurrence of anti-social behavior, which in the more serious cases leads to delinquency.

\section{Objective}

To know the relationship between family environment, supervision and the seriousness of anti-social behavior in juvenile offenders confined in a state penitentiary from Morelos.

\section{Method}

A survey was used to collect data from a population of 86 juvenile male offenders from a state penitentiary located in Morelos.

\section{Results}

Parent-children communication and support are interrelated and form the familiar environment. Thus, children's communication predicts proper supervision and monitoring, specifically in the area of knowing children's activities, which, at the same time, predict less serious anti-social behavior.

\section{Discussion and conclusion}

It is important that families have an environment based on communication and support. It is also relevant to encourage adolescents to freely communicate with their parents so supervision and monitoring become common elements that help adolescents to stay away from behaviors that could lead them to crime.

Key words: Anti-social behavior, juvenile offenders, familiar environment, parental monitoring, state of Morelos.

\section{RESUMEN}

\section{Introducción}

La supervisión parental comprende: cuidado de los hijos, conocimiento de su paradero y actividades y adaptación. Para que ésta se dé es necesario que exista comunicación y apoyo entre padres e hijos, de manera que el ambiente familiar sea agradable. Por otro lado, se ha encontrado que la inconsistencia o ausencia de ésta es un factor de riesgo de la conducta antisocial y, en casos más graves, de delincuencia en los hijos.

\section{Objetivo}

Conocer cuál es la relación que existe entre el ambiente familiar, la supervisión parental y la gravedad de la conducta antisocial en menores infractores.

\section{Método}

La población estuvo conformada por menores infractores de un Centro Tutelar del Estado de Morelos. El instrumento se aplicó a 86 hombres.

\section{Resultados}

La comunicación del hijo fue un predictor de: 1. el manejo de reglas y conocimiento de pares, y 2 . el conocimiento de las actividades del hijo. Esto último es lo que predice de manera significativa la gravedad de la conducta antisocial.

\section{Discusión y conclusión}

De acuerdo con los hallazgos, es importante que exista un ambiente familiar basado en la comunicación y el apoyo, que fomente una comunicación más libre de los adolescentes con sus padres, de manera que la supervisión parental se consiga más fácilmente. Esto ayudará para que los jóvenes no se involucren en comportamientos que los puedan llevar a delinquir.

Palabras clave: Conducta antisocial, menores infractores, ambiente familiar, supervisión parental, Estado de Morelos.

Dirección de Investigaciones Epidemiológicas y Psicosociales del Instituto Nacional de Psiquiatría Ramón de la Fuente Muñiz.

2 Dirección General del Instituto Nacional de Psiquiatría Ramón de la Fuente Muñiz.

Correspondencia: Francisco Juárez García. Dirección de Investigaciones Epidemiológicas y Psicosociales. Instituto Nacional de Psiquiatría Ramón de la Fuente Muñiz, Calz. México-Xochimilco 101, San Lorenzo Huipulco, Tlalpan, 14370, México, DF. E-mail: pacojg@imp.edu.mx

Recibido primera versión: 18 de marzo de 2014. Segunda versión: 9 de febrero de 2015. Tercera versión: 9 de julio de 2015. Aceptado: 5 de octubre de 2015. 


\section{INTRODUCCIÓN}

En nuestro país se ha incrementado el número de adolescentes que cometen conductas antisociales, así como la gravedad y la violencia de las mismas. ${ }^{1-4}$

En la ciudad de Cuernavaca, en el Estado de Morelos, el número de menores infractores* ha registrado un notable aumento. Para la Dirección de Prevención del Delito de la Secretaría de Seguridad Pública Metropolitana, esta situación puede extenderse hasta convertirse en un verdadero problema social. Las cifras revelan que en 2005 la proliferación de menores infractores fue de 30\%, tan sólo en el primer cuadro de la capital, sin tomar en cuenta las zonas aledañas. $^{5}$

La conducta antisocial hace referencia a una diversidad de actos que violan las normas sociales y los derechos de los demás. El que una conducta se incluya como antisocial depende de juicios acerca de su gravedad y su alejamiento de las pautas normativas, en función de la edad del niño, el sexo, la clase social y otras consideraciones. ${ }^{6}$ La conducta antisocial en la adolescencia consiste en actos que pueden violar la ley y que implican infracciones: fugas del hogar, asaltos, violación, asesinato, robos, destrucción de la propiedad ajena y violación grave de normas y reglas. ${ }^{6-8}$

La familia tiene un gran peso en el desarrollo infantil, y la calidad de la relación padres e hijos es de las primeras experiencias que influyen positiva o negativamente en el niño. ${ }^{7}$ Además, los medios de socialización (familia, escuela y grupo de pares, entre otros) cumplen un papel importante en el involucramiento con, o protección de, conductas problemáticas en la adolescencia, incluyendo el consumo de drogas, las infracciones a la ley llevadas a cabo por menores y el comportamiento delictivo. ${ }^{10}$

Cualquiera que sea la organización familiar, los contactos entre sus miembros o su relación con la comunidad, la disminución de la autoridad familiar, tanto moral como emocional en la vida del adolescente, implica una baja en la supervisión parental, lo que aumenta la probabilidad de conductas antisociales y delincuencia. ${ }^{11,12}$

Stattin y Kerr ${ }^{12}$ definen la supervisión parental como el conjunto de conductas que comprenden el cuidado de los hijos, el conocimiento verdadero de su paradero, sus actividades y la adaptación de las actividades fuera de casa, y tiene que ver con los esfuerzos de los padres para averiguar qué están haciendo sus hijos por medio de la solicitud y el control. Por otro lado, está el monitoreo que depende de la descripción voluntaria que hacen los chicos a sus padres acerca de las actividades que llevan a cabo en su tiempo libre, es decir, la comunicación y la disposición espontánea de los adolescentes para proporcionarles información. ${ }^{13,14}$

* Adolescentes de entre 11 y 17 años 11 meses de edad, que han infringido las leyes penales y son detenidos por la policía por tales actos, pasando por un procedimiento jurídico dependiendo de las leyes de su Estado. ${ }^{9}$
Barnes y Farrell ${ }^{15}$ indican que un fuerte apoyo parental y un alto nivel de supervisión son los factores clave en la prevención del abuso de alcohol y de otras conductas problemáticas o antisociales en adolescentes, entendiéndose "apoyo" como conductas que muestran los padres hacia los hijos, como elogiar, animar y dar afecto (físico), lo que indica al adolescente que es aceptado y amado.

Para que la supervisión parental se efectúe, es necesario que exista comunicación y apoyo entre padres e hijos, con un ambiente familiar agradable. El ambiente familiar es consecuencia de las aportaciones de todos los miembros de la familia y en especial de los padres. Los que integran la familia crean el ambiente y pueden modificarlo. De la misma manera, éste tiene la capacidad de modificar las conductas erróneas de los miembros y de potenciar al máximo las que se consideran correctas. ${ }^{16}$

La comunicación es fundamental para que el ambiente familiar se desenvuelva de manera adecuada y se tenga un clima de fraternidad y respeto al interior de la familia. La comunicación se guía por los sentimientos y la información que se transmite y se comprende. Cuando existe comunicación en una familia, es más probable que se presente compañerismo, complicidad y ambiente de unión y afecto en la casa. De este modo habrá sobre todo respeto mutuo y valores más asentados. ${ }^{17}$

Otro aspecto importante es el apoyo dentro del núcleo familiar. Éste se basa en la idea de la necesidad y obligación de proteger, resguardar, educar, comprender y dar todo tipo de satisfactores materiales y emocionales, así como de enseñar las convenciones sociales a los miembros que conforman la familia. ${ }^{17}$

Una buena relación entre padres e hijos, basada en la comunicación y el apoyo, debe operar preventivamente y ser un proceso bidireccional, incluyendo tanto la solicitud de los padres del conocimiento y control de las conductas de sus hijos, como la disposición de los hijos para hacer a sus padres parte de sus vidas. ${ }^{12}$

De acuerdo con lo revisado, la presente investigación se llevó a cabo para conocer cuál es la relación que existe entre el ambiente familiar y la supervisión parental como predictores de la gravedad de la conducta antisocial en menores infractores de un Centro Tutelar del Estado de Morelos, México.

\section{MÉTODO}

\section{Población y muestra}

Se trabajó con todos los menores infractores de un Centro Tutelar del Estado de Morelos, México. La muestra estuvo compuesta por 86 menores varones de entre 12 y 21 años de edad, con un promedio de edad de 16.4 años y una desviación estándar de 1.6 años. 


\section{Instrumento}

Se utilizó un cuestionario autoaplicable que consideró diversas áreas de problemáticas en los adolescentes. Se había validado previamente y sus principales indicadores se han mantenido en diversas encuestas. ${ }^{18,19}$

Las secciones que se utilizaron para este estudio son:

1. Escala de conducta antisocial: consta de 12 reactivos acerca del tipo de conductas antisociales que realizaron los adolescentes en el último año, por medio de preguntas como: tomar un auto sin permiso del dueño, tomar dinero o cosas, participar en riñas, golpear o herir a alguien a propósito, vender droga y usar un arma para dañar a alguien o quitarle sus pertenencias. ${ }^{18}$

La escala fue clasificada originalmente en dos factores: robos y participación en riñas, por un lado, así como comportamientos con consecuencias más graves, por el otro. Para el presente estudio se requería evaluar los diferentes niveles de gravedad de cada uno de los comportamientos, por lo que se llevó a cabo una validación por jueces expertos por medio del método de pares comparados. ${ }^{20}$ Para ello se organizaron todos los reactivos por pares, generando todas las combinaciones de parejas posibles. Se pidió a 12 jueces expertos: psiquiatras, psicólogos e investigadores de la conducta antisocial en adolescentes, que indicaran cuál era el comportamiento que consideraban más problemático en adolescentes de cada par de conductas que se les presentaba, por medio de la siguiente instrucción:

A continuación se presentan preguntas relacionadas a comportamientos de los adolescentes organizadas por pares, para cada pareja de preguntas, marque con una " $X$ " la que considere la conducta más problemática de las 2.

A cada juez se le presentó cada una de las posibles combinaciones de pares de reactivos para que señalara el que consideraba más problemático de cada par. Posteriormente, se capturaron en una matriz con las frecuencias en que fue elegido cada reactivo más problemático que su par. Una matriz $p$, en que se convirtieron las frecuencias a probabilidades de ser elegidos antes que el otro, y una matriz $z$, en que las probabilidades fueron sustituidas por su equivalente en calificación z. De esta matriz derivaron las calificaciones por reactivo utilizadas en el presente estudio, asignándole el valor 0 a no haber llevado a cabo alguna conducta antisocial hasta el valor 10, que fue indicado como el máximo nivel de gravedad (cuadro 1).

2. Ambiente familiar: incluye las subescalas de comunicación y apoyo, constituidas por 12 reactivos validados en población escolar mexicana. ${ }^{19}$ En dichas subescalas se obtuvieron cargas factoriales superiores a $0.50 \mathrm{y}$ se muestran correlaciones superiores a 0.80 en cada una de las áreas de comunicación y apoyo. ${ }^{19}$ Los factores utilizados en el presente estudio se componen de la siguiente manera:

a) Comunicación del hijo (3 reactivos).

b) Apoyo de los padres (3 reactivos).

c) Comunicación de los padres (3 reactivos).

d) Apoyo cotidiano del hijo (3 reactivos).

3. Escala de supervisión: Consta de 15 reactivos que exploran las formas de supervisión que realizan los padres, así como el interés que tienen en las actividades de sus hijos. También se les pregunta a los adolescentes si siguen las reglas que facilitan a los padres la supervisión. Se obtuvo la validez de esta escala mediante un análisis de conglomerados jerárquicos de reactivos para conocer cómo se organizaban, y para obtener la confiabilidad se utilizó el coeficiente de consistencia interna Kuder Richardson 20. Los reactivos se agruparon en dos factores: el primero se denominó "Reglas y conocimiento de los

Cuadro 1. Niveles de gravedad de los reactivos de la Escala de Conducta Antisocial

\begin{tabular}{lc}
\hline & Calificación \\
\hline 0. Ninguna conducta antisocial. & 0.00 \\
2. Tomar dinero o cosas con valor de $\$ 50$ o menos que no te pertenecen. & 1.79 \\
5. Tomar mercancía de una tienda sin pagarla (sin causar daños). & 2.84 \\
3. Tomar dinero o cosas con valor de $\$ 500$ o más que no te pertenecen. & 3.76 \\
6. Golpear o dañar algo (objeto o propiedad) que no te pertenece. & 4.32 \\
1. Tomar un auto sin permiso del dueño. & 4.35 \\
10. Tomar parte en riñas o peleas. & 4.44 \\
4. Forzar cerraduras para entrar a algún lugar que no sea tu casa. & 5.56 \\
11. Prender fuego a propósito a objetos que no te pertenecen. & 6.21 \\
8. Vender drogas (como mariguana, cocaína, etc.). & 6.83 \\
9. Golpear o herir a propósito a alguien, sin contar los pleitos o discusiones con tus hermanos. & 7.68 \\
12. Usar un cuchillo o pistola para obtener algún objeto de otra persona. & 9.21 \\
7. Atacar a alguien, usando algún objeto como: arma de fuego, cuchillo, palo, navaja, etc. & 10.00 \\
\hline
\end{tabular}


Cuadro 2. Escala de supervisión parental

\begin{tabular}{|c|c|}
\hline Reglas y conocimiento de los pares $(K R 20=0.7275)$ & Conglomerado \\
\hline c. ¿Conocen tus papás a los amigos con quién te juntas? & 1 \\
\hline k. ¿ंTus padres se preocupan acerca de las actividades que realizas en la escuela? & 1 \\
\hline I. ¿ंTus padres te han fijado las reglas acerca de con quién pasas tu tiempo libre? & 1 \\
\hline m. ¿ंTus padres te han puesto las reglas acerca de dónde puedes pasar tu tiempo libre? & 1 \\
\hline 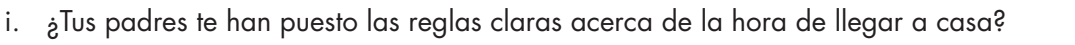 & 1 \\
\hline 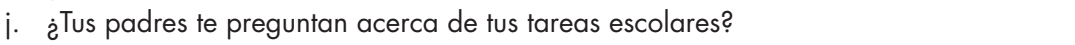 & 1 \\
\hline h. ¿̇Tus papás saben generalmente con quién acostumbras salir? & 1 \\
\hline \multicolumn{2}{|l|}{ Conocimiento de las actividades del hijo (KR20 = 0.8926) } \\
\hline n. Cuando sales, ¿le avisas a tus padres a dónde vas? & 2 \\
\hline p. Cuando sales, ¿̇le avisas a tus padres a qué hora llegas? & 2 \\
\hline o. Cuando sales, $\dot{\text { le }}$ avisas a tus padres con quién vas? & 2 \\
\hline q. Cuando sales, ¿̇llegas a la hora establecida? & 2 \\
\hline f. ¿ ¿Generalmente tus papás saben dónde te encuentras? & 2 \\
\hline 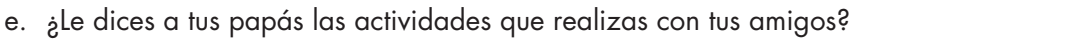 & 2 \\
\hline d. ¿̇Tus papás saben dónde viven tus amigos? & 2 \\
\hline g. ¿̇Tus papás saben generalmente los lugares a donde vas cuando sales? & 2 \\
\hline
\end{tabular}

Cuadro 3. Descripción de la muestra de hombres menores infractores del Estado de Morelos $(n=86)$

\begin{tabular}{|c|c|c|}
\hline \multirow{3}{*}{ Edad } & $\bar{X}$ & $\mathrm{~s}$ \\
\hline & 16.4 & 1.6 \\
\hline & $f$ & $\%$ \\
\hline \multicolumn{3}{|l|}{ Nivel escolar que estás cursando } \\
\hline Nunca he estudiado & 2 & 2.4 \\
\hline Primaria & 30 & 35.3 \\
\hline Secundaria & 36 & 42.4 \\
\hline Bachillerato o preparatoria & 17 & 20.0 \\
\hline \multicolumn{3}{|l|}{ Normalmente vives } \\
\hline En mi casa & 56 & 65.1 \\
\hline Parte del tiempo en casa y parte en la calle & 25 & 29.1 \\
\hline En la calle & 2 & 2.3 \\
\hline No contestó & 3 & 3.5 \\
\hline \multicolumn{3}{|l|}{ ¿Tienes papá? } \\
\hline Tengo papá & 54 & 62.8 \\
\hline Lo sustituye algún familiar & 9 & 10.5 \\
\hline No tengo papá y nadie lo sustituye & 13 & 15.1 \\
\hline Lo sustituye otro & 8 & 9.3 \\
\hline No contestó & 2 & 2.3 \\
\hline \multicolumn{3}{|l|}{ ¿Tienes mamá? } \\
\hline Tengo mamá & 75 & 87.2 \\
\hline La sustituye algún familiar & 3 & 3.5 \\
\hline No tengo mamá y nadie la sustituye & 4 & 4.7 \\
\hline La sustituye otro & 1 & 1.2 \\
\hline No contestó & 3 & 3.5 \\
\hline \multicolumn{3}{|l|}{ Actualmente vives con: } \\
\hline Con ninguno de mis papás & 15 & 17.4 \\
\hline Sólo con mi mamá & 28 & 32.6 \\
\hline Sólo con mi papá & 2 & 2.3 \\
\hline Parte del tiempo con papá y parte con mamá & 5 & 5.8 \\
\hline Con mi mamá y mi papá & 24 & 27.9 \\
\hline Con mi mamá y su pareja actual & 8 & 9.3 \\
\hline Con mi papá y su pareja actual & 3 & 3.5 \\
\hline No contestó & 1 & 1.2 \\
\hline
\end{tabular}

pares", el cual mostró una confiabilidad KR20 = 0.73; el segundo, se llamó "Conocimiento de las actividades del hijo" y obtuvo una confiabilidad KR20 $=0.89$. La confiabilidad total de la escala fue de KR20 = 0.89 (cuadro 2).

\section{Procedimiento}

Se aplicó el cuestionario a 86 hombres menores infractores de un Centro Tutelar de Morelos.

La investigación fue aprobada por los comités de ética e investigación del Instituto Nacional de Psiquiatría Ramón de la Fuente Muñiz. También fue aprobada y financiada por el Consejo Nacional de Ciencia y Tecnología por medio del convenio CONACYT-P-42273-H.

Las autoridades del Centro fungen como tutores temporales de los menores durante su estancia en el mismo, por lo que fueron ellos los que proporcionaron las facilidades de acceso a las instalaciones y el consentimiento para realizar las entrevistas a los menores. Los entrevistadores acudieron a dos salones de clase y se preguntó a los adolescentes si aceptaban responder un cuestionario, y así se obtuvo su asentimiento de forma verbal. Se les garantizó que las respuestas serían confidenciales y que no afectarían de manera positiva o negativa su proceso legal. A los menores que tenían dificultades de lecto-escritura, se les realizó la entrevista personalmente. No hubo rechazos. La duración promedio de aplicación por grupo fue de 60 minutos.

El análisis estadístico de datos se llevó a cabo mediante el paquete estadístico SPSS versión $20 .{ }^{21}$ Se obtuvieron medias y desviaciones estándar de los datos sociodemográficos, de las escalas de ambiente familiar y de supervisión, así como los coeficientes de correlación Pearson de las variables de comunicación, apoyo y supervisión parental. Así mismo, 
Cuadro 4. Calificaciones en la escala de supervisión, comunicación y apoyo de hombres menores infractores del Estado de Morelos $(n=86)$

\begin{tabular}{lcc}
\hline Escala (Puntajes mínimo-máximo posibles) & $\overline{\mathrm{X}}$ & $\mathrm{s}$ \\
\hline Gravedad de la conducta antisocial (0-10) & 4.7 & 4.2 \\
Ambiente familiar & & \\
Comunicación del hijo (1-4) & 2.5 & .9 \\
Apoyo de los padres (1-4) & 3.0 & .9 \\
Comunicación de los padres (1-4) & 2.6 & .9 \\
Apoyo significativo del hijo (1-4) & 3.0 & .8 \\
Supervisión parental & & \\
Manejo de reglas y conocimiento de las perso- & 5.5 & 1.7 \\
$\quad$ nas con quien sale el hijo (0-7) & & \\
Conocimiento de las actividades del hijo (0-8) & 5.1 & 2.9 \\
Total (0-15) & 10.6 & 4.3 \\
\hline
\end{tabular}

se realizaron regresiones lineales para establecer las relaciones entre el ambiente familiar, la supervisión y la gravedad de la conducta antisocial.

\section{RESULTADOS}

\section{Descripción de la muestra}

La mayoría de los menores (42.4\%) estaba cursando la secundaria. Antes de su ingreso al centro tutelar, más de la mitad (65.1\%) residía la mayor parte del tiempo en su casa; casi la tercera parte $(29.1 \%)$ vivía parte del tiempo en casa y parte del tiempo en la calle. El 62.8\% tenía padre, y 15.1\% mencionó no tenerlo y que nadie lo sustituía. Por otro lado, el $87.2 \%$, mencionó tener madre. El 32.6\% vivía solo con ella, el $27.9 \%$ con ambos padres y el $17.4 \%$ no vivía con alguno de ellos (cuadro 3).

En lo referente a los actos antisociales en el último año, el 59.3\% reportó haber incurrido en violencia y robos, y el $51.2 \%$ dijo haber cometido actos antisociales graves. Cabe resaltar que el $38.4 \%$ mencionó que alguno de sus familiares había tenido problemas con la policía; el 47.7\% reportó que alguno de sus familiares había estado detenido, y el $20.9 \%$ informó que alguno de sus familiares estaba detenido en el momento en que se realizó la investigación.

El promedio de gravedad de los actos antisociales fue de 4.7 (de una calificación máxima de 10). En las subescalas de ambiente familiar: "Apoyo de los padres" y "Apoyo significativo del hijo" se obtuvo una media de 3.0, de un máximo de 4 puntos posibles. De la escala de Supervisión, el factor denominado "Reglas y conocimiento de los pares" obtuvo una media de 5.5, de un máximo de 7 . La escala total de Supervisión tuvo una $\bar{X}=10.6$ de un total máximo de 15 (cuadro 4).

\section{Relación entre supervisión, comunicación y apoyo de la familia y la gravedad de la conducta antisocial}

Se obtuvieron los coeficientes de producto-momento de Pearson, y se encontró que las variables de Apoyo de los padres, Apoyo de los hijos, Comunicación de los padres y Comunicación de los hijos se relacionaron de manera significativa entre sí. Además de ello, se relacionaron con la variable de "Conocimiento de las actividades del hijo", que forma parte de la escala de supervisión (cuadro 5).

Por su parte, la Comunicación del hijo, el manejo de Reglas, el conocimiento de los pares y el conocimiento de las actividades del hijo se relacionaron significativamente con la gravedad de la conducta antisocial. Dicha relación fue negativa o inversa, es decir, que, a mayor comunicación del hijo, a mejor manejo de reglas y conocimiento de las actividades del hijo, menor fue la gravedad de la conducta antisocial (cuadro 5).

\section{Predictores de la supervisión con la gravedad de la conducta antisocial}

Se llevaron a cabo diversos modelos de regresión lineal para evaluar cómo se relacionaban las áreas del ambiente familiar, la supervisión parental y la gravedad de la conducta antisocial. El objetivo de la primera regresión lineal fue conocer la relación de los cuatro factores del ambiente familiar

Cuadro 5. Relación entre supervisión, ambiente familiar y la gravedad de la conducta antisocial en menores infractores del Estado de Morelos $(n=86)$

\begin{tabular}{|c|c|c|c|c|c|c|}
\hline & $\begin{array}{c}\text { Gravedad } \\
\text { de la conducta } \\
\text { antisocial }\end{array}$ & $\begin{array}{l}\text { Comunicación } \\
\text { del hijo }\end{array}$ & $\begin{array}{c}\text { Apoyo } \\
\text { de los padres }\end{array}$ & $\begin{array}{l}\text { Comunicación } \\
\text { de los padres }\end{array}$ & $\begin{array}{l}\text { Apoyo signifi- } \\
\text { cativo del hijo }\end{array}$ & $\begin{array}{c}\text { Manejo de reglas } \\
\text { y conocimiento } \\
\text { de los pares }\end{array}$ \\
\hline Comunicación del hijo & $-0.329 *$ & & & & & \\
\hline Apoyo de los padres & -0.182 & $0.660 *$ & & & & \\
\hline Comunicación de los padres & -0.128 & 0.721 * & $0.709 *$ & & & \\
\hline Apoyo significativo del hijo & -0.120 & $0.613^{*}$ & $0.657^{*}$ & $0.589 *$ & & \\
\hline $\begin{array}{l}\text { Establecimiento de reglas y conocimiento } \\
\text { de los pares }\end{array}$ & $-0.321^{*}$ & $0.358 *$ & $0.268 * *$ & 0.210 & 0.171 & \\
\hline Conocimiento de las actividades del hijo & $-0.585^{*}$ & 0.484 * & $0.306^{*}$ & $0.315^{*}$ & $0.326 *$ & 0.661 * \\
\hline
\end{tabular}

Correlación de Pearson, ${ }^{*} p<.01,{ }^{* *} p<.05$ 


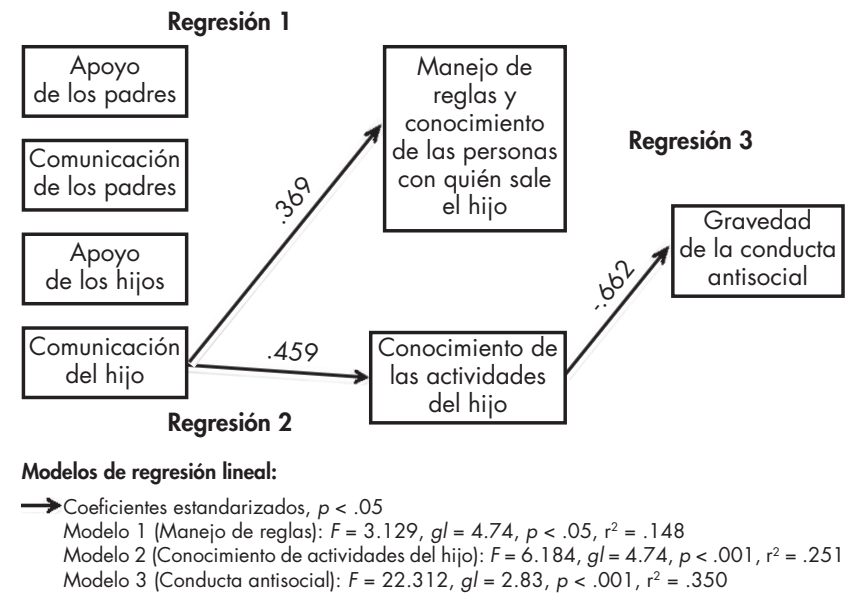

Figura 1. Relación entre conducta antisocial, comunicación y apoyo en la familia y supervisión parental en menores infractores del Estado de Morelos.

con el factor de supervisión parental denominado "Reglas y conocimiento de los pares". La segunda se realizó con el objetivo de comprender cómo se relacionaban los cuatro factores del ambiente familiar con el factor llamado "Conocimiento de las actividades del hijo". Con la tercera y última regresiones lineales se deseaba conocer cómo se relacionaban los dos factores que conforman la Supervisión parental con la gravedad de la conducta antisocial.

En la primera regresión lineal se encontró que la comunicación del hijo predijo de manera significativa el manejo de reglas y el conocimiento de las personas con las que sale el hijo. En la segunda resultó que la comunicación del hijo también predijo de manera significativa el conocimiento de sus actividades. Finalmente, en la tercera, el conocimiento de las actividades del hijo predijo de manera inversa la gravedad de la conducta antisocial; esto es, que cuanta mayor supervisión parental, existe una menor gravedad en la conducta antisocial (figura 1).

A pesar de que la comunicación de los padres, el apoyo de los hijos, el apoyo de los padres y la comunicación de los hijos se relacionan entre sí y conforman el ambiente familiar, sólo resultó significativa la relación entre la comunicación del hijo y el manejo de reglas y el conocimiento de las personas con las que sale el hijo y el conocimiento de sus actividades (figura 1).

\section{DISCUSIÓN Y CONCLUSIÓN}

Medina ${ }^{17}$ menciona que la familia no sólo significa una agrupación de personas, o una estructura, sino una unión complementaria, rica en vínculos afectivos de distinto tipo y en diversas direcciones. La importancia de la familia en la educación de los hijos es fundamental, ya que esta agrupación es la que proporciona las herramientas, así como el criterio para que los adolescentes aprendan a protegerse de situaciones y personas que pueden dañarlos. En ese sentido, la calidad del ambiente familiar y de las relaciones familiares no depende necesariamente de la estructura familiar, ni de con quién viven los adolescentes, sino más bien de la facilidad de los padres para relacionarse, comunicarse y apoyar a sus hijos en los momentos importantes, lo cual genera un ambiente familiar sano y propicio para que se realice la supervisión.

La comunicación y el apoyo de padres e hijos se relacionaron entre sí y conformaron el ambiente familiar. La comunicación del hijo predijo una supervisión adecuada específicamente en el área del conocimiento de sus actividades y ésta predijo a su vez una menor gravedad de la conducta antisocial para esta muestra de menores infractores, lo cual coincide con la postura de Dishion y McMahon, ${ }^{13}$ quienes proponen que la buena comunicación y las habilidades para la solución de problemas pueden servir para mantener una sana relación entre padres y adolescentes, así como para supervisar a los hijos en sus actividades diarias.

Considerando lo que Dishion y McMahon ${ }^{13}$ proponen en relación con que el monitoreo puede servir como un factor de protección para los niños y jóvenes que viven en ambientes de alto riesgo, la comunicación del hijo predijo una supervisión adecuada específicamente en el área del conocimiento de las actividades de éste, lo que a su vez predijo una menor gravedad de la conducta antisocial.

De acuerdo con estos hallazgos, es necesario estimular el apoyo y la comunicación en el ambiente familiar y fomentar que los adolescentes se comuniquen libremente con sus padres, de manera que la supervisión parental se realice más fácilmente para ayudar a que estos menores tengan más herramientas que les ayuden a no reincidir $\mathrm{y}$, al hablar de los adolescentes en general, para que no se involucren en actos antisociales.

Por otro lado, es importante mencionar que un alto porcentaje de estos jóvenes tienen familiares que habían sido detenidos o estaban detenidos al momento en que se realizó la encuesta. Esto es un punto fundamental, ya que diversos teóricos señalan que los ejemplos criminógenos en el hogar son muy significativos en el desarrollo de conductas antisociales en los adolescentes. ${ }^{6,7}$ Sin embargo, aún faltan estudios al respecto, por lo que deben realizarse futuras investigaciones en las que se analice la asociación entre la conducta infractora, su gravedad y la relación con familiares detenidos, además de identificar cómo afecta esta relación al ambiente familiar y a las acciones de supervisión de los hijos.

Una limitación del presente estudio es que se realizó con un instrumento de preguntas cerradas, por lo que se debería hacer una futura investigación exhaustiva en la que se indaguen las formas de interacción de las familias de los menores infractores. Además, sería importante tomar en cuenta no sólo la percepción de los adolescentes de la supervisión parental, sino también saber la opinión de los 
padres y conocer cuáles son sus estrategias de supervisión y si ambas partes las consideran útiles.

La presente investigación se realizó únicamente con menores infractores. Sería importante contar con acercamientos a menores infractores y no infractores, con la finalidad de observar el fenómeno en distintas poblaciones y enriquecer los hallazgos obtenidos. Asimismo, sería importante realizar investigaciones con mujeres menores infractoras y contrastar los resultados para identificar si existen diferencias en las estructuras y relaciones familiares para poder incidir con ellas de forma adecuada.

A pesar de las limitaciones, el presente trabajo es de gran relevancia al aportar información sobre una población de difícil acceso, lo cual permitió contar con un panorama de su situación particular para proponer acciones de prevención y tratamiento acordes con sus necesidades.

Esta población es estigmatizada por vivir en una situación de conflicto con la ley. Abordarlos desde una visión objetiva, de cómo perciben su entorno familiar, permite que los diferentes sectores que tienen contacto con ellos puedan incidir en la forma en que se aborda el trabajo con este grupo y sobre todo en la elaboración de programas de prevención indicada que incluyan las condiciones que estos jóvenes requieren para un mejor retorno a su seno familiar. Los programas también deben ayudar a brindar estrategias a las familias para la adecuada supervisión de sus hijos, protegiéndolos de no reincidir en la comisión de delitos, y que ellos, así como aquellos adolescentes que no llevan a cabo este tipo de comportamientos, se mantengan alejados de llevar a cabo conductas de riesgo en general.

\section{Financiamiento}

El presente trabajo se realizó gracias al financiamiento del Consejo Nacional de Ciencia y Tecnología a través del convenio 42273-H para el proyecto del Instituto Nacional de Psiquiatría: 4216 "Detección de necesidades de menores infractores del Distrito Federal".

\section{Conflictos de interés}

Los autores declaran no tener conflictos de interés.

\section{Agradecimientos}

Al psicólogo José Alberto Jiménez Tapia por su apoyo para la elaboración de este artículo.

\section{REFERENCIAS}

1. Consejo de Menores. Memoria 1996-mayo 1997. México: Secretaría de Gobernación; 1997.
2. Consejo de Menores: Memoria 1996-1999. México: Secretaría de Gobernación; 2000.

3. Juárez F, Villatoro J, Gutiérrez ML, Fleiz C et al. Tendencias de la conducta antisocial en estudiantes del Distrito Federal: Mediciones 19972003. Salud Ment 2005;28(3):60-68.

4. Secretaría de Seguridad Pública. Consejo de Menores, 2003. Sitio Internet http://www.ssp.gob.mx/application?pageid=cmenores_sub_2\&docId=838 Fecha de último acceso: 25/07/2005.

5. CUEVAS E: Se incrementa número de los menores infractores. Jornada Morelos, 5 de julio; 2005.

6. Kazdin A. Tratamiento de la conducta antisocial en la infancia y la adolescencia. Barcelona, España: Martínez-Roca; 1988.

7. Alcántara E: Menores con conducta antisocial. México. Porrúa-Universidad Anáhuac; 2001.

8. Craig G, Baucom D. Desarrollo psicológico. México. Pearson; 2001.

9. Diario Oficial de la Federación. Ley para el Tratamiento de Menores Infractores para el Distrito Federal en Materia Común y para Toda la República en Materia Federal, 1991, feb. En: InfoJus, sistema de consulta del Instituto de Investigaciones Jurídicas de la Universidad Nacional Autónoma de México. Legislación Federal (vigente al 3/02/2015). Sitio internet: http://info4.juridicas.unam.mx/ijure/tcfed/186.htm?s= Fecha de último acceso: 5/02/2015.

10. Juárez F. Predictores de la conducta antisocial y su relación con el uso de drogas en una muestra nacional de estudiantes de enseñanza media y media superior. Tesis de licenciatura. México: Facultad de Psicología, UNAM; 1999.

11. De la Garza F, De la Vega B, Zúñiga V, Villarreal R. La cultura del menor infractor. México: Trillas; 1987.

12. Stattin H, Kerr M. Parental monitoring: A reinterpretation. Child Development 2000;71(4):1072-1085.

13. Dishion T, McMahon R. Parental monitoring and the prevention of child and adolescent problem behavior: A conceptual and empirical formulation. Clinical Child Family Psychology Review 1998;1(1):6175.

14. Patterson G, Stouthamer-Loeber M. The correlation of family management practices and delinquency. Child Development 1984;55(4):12991307.

15. Barnes G, Farrell M. Parental support and control as predictors of adolescent drinking, delinquency, and related problem behaviors. J Marriage Family 1992;54:763-776.

16. Villalobos M. La relación familiar, algunos de sus efectos perturbadores en la organización del sujeto. Cuadernos Psicología-Colombia 1994;13(2):7-24.

17. Medina $V$ (ed.). La comunicación entre padres e hijos. En: Guía Infantil.com, (s/f), revista en línea: http://www.guiainfantil.com/educacion/ familia/comfamilia.htm. Fecha de último acceso: 5/02/2015

18. Juárez F, Medina-Mora ME, Berenzon S, Villatoro J et al. Antisocial behavior: Its relation to selected sociodemographic variables and alcohol and drug use among mexican students. Substance Use Misuse 1998;33(7):1437-1459.

19. Villatoro J, Andrade-Palos $P$, Fleiz C, Medina-Mora ME et al. La relación padres-hijos: una escala para evaluar el ambiente familiar en adolescentes. Salud Ment 1997;20(2):21-27.

20. Edwards AL. Techniques of Attitude Scale Construction. Nueva York:, Appleton Century Crofts; 1957.

21. IBM SPSS Statistics versión 20 [Software de computadora]. New York: IBM Corporation, SPSS Inc.; 2010. 